Article

\title{
Existence and Uniqueness of Zeros for Vector-Valued Functions with K-Adjustability Convexity and Their Applications
}

\author{
Wei-Shih Du \\ Department of Mathematics, National Kaohsiung Normal University, Kaohsiung 82444, Taiwan; \\ wsdu@mail.nknu.edu.tw
}

Received: 28 June 2019; Accepted: 24 August 2019; Published: 2 September 2019

Abstract: In this paper, we introduce the new concepts of $K$-adjustability convexity and strictly $K$-adjustability convexity which respectively generalize and extend the concepts of $K$-convexity and strictly $K$-convexity. We establish some new existence and uniqueness theorems of zeros for vector-valued functions with $K$-adjustability convexity. As their applications, we obtain existence theorems for the minimization problem and fixed point problem which are original and quite different from the known results in the existing literature.

Keywords: K-convexity; strictly $\mathrm{K}$-convexity; $\mathrm{K}$-adjustability convexity; strictly $\mathrm{K}$-adjustability convexity; nonlinear scalarization function; $(e, K)$-lower semicontinuous; zero for a vector-valued function; minimization problem; fixed point problem

MSC: 26B25; 47H10; 65K10

\section{Introduction and Preliminaries}

It is well known that convex analysis has played an important role in almost all branches of mathematics, physics, economics, and engineering. Convexity is an ancient and natural notion and the theory of convex functions is an essential part of the general subject of convexity.

Let $V$ be a vector space. A nonempty subset $A$ of $V$ is called convex if for any $x, y \in A, \lambda x+(1-$ $\lambda) y \in A$ for all $\lambda \in[0,1]$. Let $X$ be a nonempty convex subset of $V$. A real-valued function $f: X \rightarrow \mathbb{R}$ is called convex if

$$
f(t x+(1-t) y) \leq t f(x)+(1-t) f(y)
$$

for all $x, y \in X$ and $t \in[0,1]$. If the above inequality (1) is strict whenever $x \neq y$ and $0<t<1$, then $f$ is called strictly convex. A function $f: X \rightarrow \mathbb{R}$ is called concave (resp. strictly concave) if $-f$ is convex (resp. strictly convex). A large amount of new notions of generalized convexity and concavity have been investigated by several authors; see, for example, ref. [1-15] and references therein.

The general vector optimization problem $(V O P)$ for a vector-valued function $f: X \rightarrow V_{2}$ can be formalized as follows:

$$
(V O P)\left\{\begin{array}{l}
\text { Optimize } f(x), \\
\text { subject to } x \in X
\end{array},\right.
$$

where $V_{1}$ and $V_{2}$ be vector spaces and $X$ is a nonempty subset of $V_{1}$. Vector optimization problems have been intensively investigated, and various feasible methods have been proposed over a century and has made more important contributions to improve our understanding of the real world around us in various fields. Convex analysis and vector optimization has wide and significant applications in many areas of mathematics, including nonlinear analysis, finance mathematics, vector differential equations and inclusions, dynamic system theory, control theory, economics, game theory, machine 
learning, multiobjective programming, multi-criteria decision making, game theory, signal processing, and so forth. For more details, see, e.g., ref. [1,7-10,16] and references therein.

In reality, we often encounter non-convex functions or non-concave functions when solving problems in the real world, so these known results for convex functions or concave functions are not easily applicable to work. Motivated by that reason, in this paper, we study and introduce the new concepts of $K$-adjustability convexity and strictly $K$-adjustability convexity (see Definition 1 below). A nontrivial example is given to illustrate that the concept of K-adjustability convexity is a real generalization of the concept of $K$-convexity. In Section 3, we establish some new existence and uniqueness theorems of zeros for vector-valued functions with $K$-adjustability convexity. As their applications, we obtain existence theorems for minimization problem and fixed point problem which are original and quite different from the known results in the literature.

\section{New Concepts of K-Adjustability Convexity and Strictly K-Adjustability Convexity}

Let $V$ be a topological vector space (t.v.s., for short) with its zero vector $\theta_{V}$. Let $A$ be a nonempty subset of $V$. We use the notations $\bar{A}, c o(A)$ and $\overline{c o}(A)$ to denote the closure, convex hull and closed convex hull (i.e., the closure of the convex hull) of $A$, respectively. A nonempty subset $K$ of $V$ is called a convex cone if $K+K \subseteq K$ and $\lambda K \subseteq K$ for $\lambda \geq 0$. A cone $K$ is pointed if $K \cap(-K)=\left\{\theta_{V}\right\}$. For a given cone $K \subseteq V$, we can define a partial ordering $\lesssim_{K}$ with respect to $K$ by

$$
x \precsim K y \Longleftrightarrow y-x \in K \text {. }
$$

$x \prec_{K} y$ will stand for $x \precsim_{K} y$ and $x \neq y$, while $x \ll_{K} y$ will stand for $y-x \in$ int $K$, where int $K$ denotes the interior of $K$. A function $\varphi: V \rightarrow V$ is called to be $\precsim_{K}$-nondecreasing if $x, y \in V$ with $x \precsim_{K} y$ implies $\varphi(x) \precsim_{K} \varphi(y)$.

Let $X$ be a topological space. A real-valued function $h: X \rightarrow \mathbb{R}$ is lower semicontinuous (in short lsc) (resp. upper semicontinuous, in short $u s c$ ) if $\{x \in X: h(x) \leq r\}$ (resp. $\{x \in X: h(x) \geq r\}$ ) is closed for each $r \in \mathbb{R}$.

Let $Y$ be a t.v.s. with its zero vector $\theta, K$ be a proper (i.e., $K \neq Y$ ), closed and convex pointed cone in $Y$ with $i n t K \neq \varnothing, e \in i n t K$, and $\precsim_{K}$ be a partial ordering with respect to $K$. A vector-valued function $f: X \rightarrow Y$ is said to be $(e, K)$-lower semicontinuous $[9,17]$ if for each $r \in \mathbb{R}$, the set $\{x \in X: f(x) \in$ $r e-K\}$ is closed.

In this paper, we introduce the concepts of K-adjustability convexity and strictly K-adjustability convexity.

Definition 1. Let $V_{1}$ and $V_{2}$ be vector spaces, $X$ be a nonempty convex set in $V_{1}, K$ be a given convex cone in $V_{2}$ and $\mu: V_{2} \rightarrow V_{2}$ be a mapping. A vector-valued function $f: X \rightarrow V_{2}$ is called

(i) K-adjustability convex with respect to $\mu$ (abbreviated as $(K, \mu)$-adjconvex) if

$$
\mu(t f(x)+(1-t) f(y))-f(t x+(1-t) y) \in K
$$

for all $x, y \in X$ and $t \in[0,1]$. In particular, $f$ is called $K$-convex if $\mu$ is an identity mapping on $V_{2}$ and (2) becomes

$$
t f(x)+(1-t) f(y)-f(t x+(1-t) y) \in K
$$

for all $x, y \in X$ and $t \in[0,1]$.

(ii) strictly K-adjustability convex with respect to $\mu$ (abbreviated as strictly $(K, \mu)$-adjconvex) if

$$
\mu(t f(x)+(1-t) f(y))-f(t x+(1-t) y) \in \operatorname{int} K
$$


for all $x, y \in X$ with $x \neq y$ and $t \in(0,1)$. In particular, $f$ is called strictly $K$-convex if $\mu$ is an identity mapping on $V_{2}$ and (3) becomes

$$
t f(x)+(1-t) f(y)-f(t x+(1-t) y) \in \operatorname{int} K
$$

for all $x, y \in X$ with $x \neq y$ and $t \in(0,1)$.

Here, we give an example where $f$ is $K$-adjconvex but not $K$-convex.

Example 1. Let $V_{1}=\mathbb{R}, V_{2}=\mathbb{R}^{2}, X=[-1,1]$ and $K=\mathbb{R}_{+}^{2}:=\left\{\left(x_{1}, x_{2}\right) \in \mathbb{R}^{2}: x_{i} \geq 0, i=1,2\right\}$. Then $X$ is a nonempty convex subset of $V_{1}$ and $K$ is a convex cone in $V_{2}$. Let $f: X \rightarrow V_{2}$ be defined by

$$
f(x)= \begin{cases}(-x, 0), & x \in[0,1] \\ (0, x), & x \in[-1,0)\end{cases}
$$

Take $\widehat{x}=\frac{1}{2}$ and $\widehat{y}=-\frac{1}{2}$. Thus, we get

$$
\frac{1}{2} f(\widehat{x})+\frac{1}{2} f(\widehat{y})-f\left(\frac{1}{2} \widehat{x}+\frac{1}{2} \widehat{y}\right)=\left(-\frac{1}{4},-\frac{1}{4}\right)-(0,0)=\left(-\frac{1}{4},-\frac{1}{4}\right) \notin K,
$$

which show that $f$ is not $K$-convex. Now, let $\mu: V_{2} \rightarrow V_{2}$ be defined by

$$
\mu(x, y)=(\max \{|x|,|y|\}, 0) \quad \text { for }(x, y) \in V_{2} .
$$

We claim that $f$ is $(K, \mu)$-adjconvex. Let $x, y \in X$ and $t \in[0,1]$ be given. We consider the following four possible cases:

Case 1. If $x, y \in[0,1]$, then $t f(x)+(1-t) f(y)=(a, 0)$ for some $a \leq 0$ and $f(t x+(1-t) y)=(b, 0)$ for some $b \leq 0$. Since $\max \{|a|,|0|\}-b \geq 0$, we obtain

$$
\mu(t f(x)+(1-t) f(y))-f(t x+(1-t) y)=(\max \{|a|,|0|\}-b, 0) \in K .
$$

Case 2. If $x, y \in[-1,0)$, then $t f(x)+(1-t) f(y)=(0, c)$ for some $c \leq 0$ and $f(t x+(1-t) y)=(0, d)$ for some $d<0$. So $\mu(t f(x)+(1-t) f(y))=(\max \{|0|,|c|\}, 0)$ and we get

$$
\mu(t f(x)+(1-t) f(y))-f(t x+(1-t) y) \in K .
$$

Case 3. Assume that $x \in[0,1]$ and $y \in[-1,0)$. Then $t f(x)+(1-t) f(y)=(m, n)$ for some $m, n \leq 0$.

- If $t x+(1-t) y \in[0,1]$, then $f(t x+(1-t) y)=(\lambda, 0)$ for some $\lambda \leq 0$. Hence, we have

$$
\mu(t f(x)+(1-t) f(y))-f(t x+(1-t) y)=(\max \{|m|,|n|\}-\lambda, 0) \in K .
$$

- If $t x+(1-t) y \in[-1,0)$, then $f(t x+(1-t) y)=(0, s)$ for some $s<0$. Therefore, we get

$$
\mu(t f(x)+(1-t) f(y))-f(t x+(1-t) y)=(\max \{|m|,|n|\},-s) \in K .
$$

Case 4. Assume that $x \in[-1,0)$ and $y \in[0,1]$. Following the same argument as Case 3, we can verify

$$
\mu(t f(x)+(1-t) f(y))-f(t x+(1-t) y) \in K .
$$

Therefore, by above cases, we prove that $f$ is $(K, \mu)$-adjconvex.

In Definition 1 , if we take $V=V_{1}, V_{2}=\mathbb{R}, K=[0,+\infty) \subset \mathbb{R}$, then we obtain the following concepts. 
Definition 2. Let $X$ be a nonempty convex subset of a vector space $V$ and $\mu: \mathbb{R} \rightarrow \mathbb{R}$ be a function. A real-valued function $f: X \rightarrow \mathbb{R}$ is called

(i) adjustability convex with respect to $\mu$ (abbreviated as $(\mu)$-adjconvex) if

$$
f(t x+(1-t) y) \leq \mu(t f(x)+(1-t) f(y))
$$

for all $x, y \in X$ and $t \in[0,1]$. In particular, if $\mu$ is an identity mapping on $\mathbb{R}$, then $f$ is called convex.

(ii) strictly adjustability convex with respect to $\mu$ (abbreviated as strictly $(\mu)$-adjconvex) if

$$
f(t x+(1-t) y)<\mu(t f(x)+(1-t) f(y))
$$

for all $x, y \in X$ with $x \neq y$ and $t \in(0,1)$. In particular, if $\mu$ is an identity mapping on $\mathbb{R}$, then $f$ is called strictly convex.

In the following, unless otherwise specified, we always suppose that $Y$ is a locally convex Hausdorff t.v.s. with its zero vector $\theta, K$ be a proper, closed and convex pointed cone in $Y$ with int $K \neq \varnothing, e \in \operatorname{int} K$, and $\precsim_{K}$ be a partial ordering with respect to $K$. Recall that the nonlinear scalarization function $\xi_{e}: Y \rightarrow \mathbb{R}$ is defined by

$$
\xi_{e}(y)=\inf \{r \in \mathbb{R}: y \in r e-K\}, \text { for all } y \in Y
$$

Obviously, $\xi_{e}(\theta)=0$.

The following known result is very crucial in our proofs.

Lemma 1. (see $[1,5,16,18-23])$. For each $r \in \mathbb{R}$ and $y \in Y$, the following statements are satisfied:

(i) $\xi_{e}(y) \leq r \Longleftrightarrow y \in r e-K$;

(ii) $\xi_{e}(y)>r \Longleftrightarrow y \notin r e-K$;

(iii) $\xi_{e}(y) \geq r \Longleftrightarrow y \notin r e-i n t K$;

(iv) $\xi_{e}(y)<r \Longleftrightarrow y \in r e-i n t K$;

(v) $\xi_{e}(\cdot)$ is positively homogeneous and continuous on $Y$;

(vi) if $y_{1} \in y_{2}+K\left(\right.$ i.e., $\left.y_{2} \precsim_{K} y_{1}\right)$, then $\xi_{e}\left(y_{2}\right) \leq \xi_{e}\left(y_{1}\right)$;

(vii) $\xi_{e}\left(y_{1}+y_{2}\right) \leq \xi_{e}\left(y_{1}\right)+\xi_{e}\left(y_{2}\right)$ for all $y_{1}, y_{2} \in Y$.

By Applying (i) of Lemma 1, one can easily verify the following result; see also [19,24].

Lemma 2. Let $X$ be a topological space and $f: X \rightarrow Y$ be a vector-valued function. Then $f$ is $(e, K)$-lower semicontinuous if and only if $\xi_{e} \circ f$ is lower semicontinuous.

\section{New Existence Results and Their Applications to Minimization Problem and Fixed Point Problem}

The following lemma is very important and will be used for proving our main results.

Lemma 3. Let $\mu: Y \rightarrow Y$ be a vector-valued function satisfying the following condition:

(A) For any $\epsilon>0$, there exists $\gamma>0$ such that

$$
x \notin-K \text { and } x \ll_{K} \gamma e \text { implies } \mu(x) \ll_{K} \epsilon e .
$$

Then there exists a strictly decreasing sequence $\left\{\lambda_{n}\right\}_{n \in \mathbb{N}}$ of positive real numbers such that $\mu\left(\lambda_{n+1} e\right) \ll_{K} \lambda_{n} e$ for all $n \in \mathbb{N}$ and $\lambda_{n} \downarrow 0$ as $n \rightarrow \infty$.

Proof. Given $\lambda_{1}>0$. Then, by (A), there exists $\delta_{1}>0$ such that

$$
x \notin-K \text { and } x \ll_{K} \delta_{1} e \text { implies } \mu(x) \ll_{K} \lambda_{1} e \text {. }
$$


Let $\lambda_{2}=\min \left\{\frac{\delta_{1}}{2}, \frac{\lambda_{1}}{2}\right\}$ and take $w_{1}=\lambda_{2} e \in \operatorname{int} K$. Then we have the following:

- $w_{1} \notin-K$;

- $w_{1} \ll_{K} \delta_{1} e ;$

- $\lambda_{2}<\lambda_{1}$.

So we have from (4) that

$$
\mu\left(w_{1}\right) \ll_{K} \lambda_{1} e .
$$

For $\lambda_{2}$, it must exist $\delta_{2}>0$ such that

$$
x \notin-K \text { and } x \ll_{K} \delta_{2} e \text { implies } \mu(x) \ll_{K} \lambda_{2} e .
$$

Put $\lambda_{3}=\min \left\{\frac{\delta_{2}}{2}, \frac{\lambda_{2}}{2}\right\}$ and $w_{2}=\lambda_{3} e \in \operatorname{int} K$. Thus $\lambda_{3}<\lambda_{2}$ and, by (5), we obtain

$$
\mu\left(w_{2}\right) \ll_{K} \lambda_{2} e .
$$

Continuing this process, for $\lambda_{j}, j \in \mathbb{N}$ with $j \geq 2$, it must exist $\delta_{j}>0$ such that

$$
x \notin-K \text { and } x \ll_{K} \delta_{j} e \text { implies } \mu(x) \ll_{K} \lambda_{j} e .
$$

Take

$$
\lambda_{j+1}=\min \left\{\frac{\delta_{j}}{2}, \frac{\lambda_{j}}{2}\right\}
$$

and

$$
w_{j}=\lambda_{j+1} e \in Y .
$$

Then we get from (6) and (7) that $\lambda_{j+1}<\lambda_{j}$ and $\mu\left(w_{j}\right) \ll_{K} \lambda_{j}$ e. Therefore, we can construct a strictly decreasing sequences $\left\{\lambda_{n}\right\}$ of positive real numbers such that

$$
\mu\left(\lambda_{n+1} e\right) \ll_{K} \lambda_{n} e \text { for all } n \in \mathbb{N} .
$$

By (7), we have $0<\lambda_{n+1} \leq \frac{\lambda_{1}}{2^{n}}$ for $n \in \mathbb{N}$, which yields $\lambda_{n} \downarrow 0$ as $n \rightarrow \infty$. The proof is completed.

The following result is immediate from Lemma 3 if we take $Y=\mathbb{R}, K=[0,+\infty) \subset \mathbb{R}$ and $e=1$.

Corollary 1. Let $\mu: \mathbb{R} \rightarrow \mathbb{R}$ be a function satisfying the following condition:

$\left(A_{\mathbb{R}}\right)$ For any $\epsilon>0$, there exists $c>0$ such that

$$
0<x<c \text { implies } \mu(x)<\epsilon .
$$

Then there exists a strictly decreasing sequence $\left\{\lambda_{n}\right\}_{n \in \mathbb{N}}$ of positive real numbers such that $\mu\left(\lambda_{n+1}\right)<\lambda_{n}$ for all $n \in \mathbb{N}$ and $\lambda_{n} \downarrow 0$ as $n \rightarrow \infty$.

Corollary 2. Let $\mu: \mathbb{R} \rightarrow \mathbb{R}$ be a function satisfying $\lim _{x \rightarrow 0^{+}} \mu(x)=0$. Then there exists a strictly decreasing sequence $\left\{\lambda_{n}\right\}_{n \in \mathbb{N}}$ of positive real numbers such that $\mu\left(\lambda_{n+1}\right)<\lambda_{n}$ for all $n \in \mathbb{N}$ and $\lambda_{n} \downarrow 0$ as $n \rightarrow \infty$.

Proof. For any $\epsilon>0$, since $\lim _{x \rightarrow 0^{+}} \mu(x)=0$, there exists $\delta=\delta(\epsilon)>0$ such that

$$
0<x<\delta \text { implies } \mu(x)<\epsilon .
$$

Therefore, the conclusion is immediate from Corollary 1.

We now establish the following crucial and useful existence result which is one of the main results of this paper and will be applied to minimization problem and fixed point problem. 
Theorem 1. Let $(E,\|\cdot\|)$ be a normed linear space, $Y$ be a locally convex Hausdorff t.v.s. with its zero vector $\theta, K$ be a proper, closed and convex pointed cone in $Y$ with int $K \neq \varnothing$, and let $e \in$ int $K$ be fixed. Let $W$ be a nonempty weakly compact and convex subset of $E, \mu: Y \rightarrow Y$ be a $\precsim K$-nondecreasing vector-valued function satisfying the condition $(A)$ as in Lemma 3 and $f: W \rightarrow Y$ be a vector-valued function. Assume that

(H1) for any positive real number $\gamma,\{x \in W: f(x) \in \gamma e-K\}$ is a nonempty closed subset of $W$,

(H2) $f$ is $(K, \mu)$-adjconvex.

Then there exists $v \in W$ such that $f(v) \in-K$.

Proof. By applying Lemma 3, there exists a strictly decreasing sequence $\left\{\lambda_{n}\right\}_{n \in \mathbb{N}}$ of positive real numbers such that

$$
\mu\left(\lambda_{n+1} e\right) \ll_{K} \lambda_{n} e \text { for all } n \in \mathbb{N},
$$

and $\lambda_{n} \downarrow 0$ as $n \rightarrow \infty$. For any $n \in \mathbb{N}$, let

$$
C_{n}=\left\{x \in W: f(x) \in \lambda_{n} e-K\right\} .
$$

Define $F: W \rightarrow \mathbb{R}$ by

$$
F(x)=\xi_{e} \circ f(x) \text { for } x \in W
$$

Applying Lemma 1, we have

$$
C_{n}=\left\{x \in W: F(x) \leq \lambda_{n}\right\}
$$

Thus, by (H1), $C_{n}$ is a nonempty closed subset of $W$. Clearly, $C_{n+1} \subseteq C_{n}$ for all $n \in \mathbb{N}$. We choose an arbitrary point $z_{n}$ from $C_{n}$ for all $n \in \mathbb{N}$. For any $m, n \in \mathbb{N}$ with $m \geq n$, let

$$
D_{m, n}=\left\{z_{i}: n+1 \leq i \leq m+1\right\} .
$$

We verify that

$$
\operatorname{co}\left(D_{m, n}\right) \subseteq C_{n} \quad \text { for all } m, n \in \mathbb{N} \text { with } m \geq n .
$$

Indeed, let $m, n \in \mathbb{N}$ with $m \geq n$. If $m=n$, then

$$
\operatorname{co}\left(D_{n, n}\right)=\left\{z_{n+1}\right\} \subseteq C_{n+1} \subseteq C_{n}
$$

and (9) is true. For $m \geq 2$ and $n=m-1, \operatorname{co}\left(D_{m, m-1}\right)=\operatorname{co}\left(\left\{z_{m}, z_{m+1}\right\}\right)$. If $x \in \operatorname{co}\left(D_{m, m-1}\right)$, then there exists $t \in[0,1]$ such that

$$
x=t z_{m}+(1-t) z_{m+1} .
$$

Since $z_{m}, z_{m+1} \in C_{m}, f\left(z_{m}\right), f\left(z_{m+1}\right) \in \lambda_{m} e-K$. Since $K$ is a convex cone, we get

$$
t f\left(z_{m}\right)+(1-t) f\left(z_{m+1}\right) \in \lambda_{m} e-K .
$$

Thus, there exists $\zeta \in K$ such that $t f\left(z_{m}\right)+(1-t) f\left(z_{m+1}\right)=\lambda_{m} e-\zeta$. Since $\lambda_{m} e-\zeta \precsim K \lambda_{m} e$ and $\mu$ is $\precsim K$-nondecreasing, we obtain

$$
\mu\left(t f\left(z_{m}\right)+(1-t) f\left(z_{m+1}\right)\right)=\mu\left(\lambda_{m} e-\zeta\right) \precsim K \mu\left(\lambda_{m} e\right)
$$

Taking into account (H2), (8) and (11), we get

$$
\begin{gathered}
f(x) \precsim_{K} \mu\left(t f\left(z_{m}\right)+(1-t) f\left(z_{m+1}\right)\right) \precsim_{K} \mu\left(\lambda_{m} e\right) \ll_{K} \lambda_{m-1} e \\
\Longleftrightarrow \quad F(x)=\xi_{e} \circ f(x)<\lambda_{m-1} \quad \text { (by Lemma 1) }
\end{gathered}
$$


which means that $x \in C_{m-1}$. Hence $c o\left(D_{m, m-1}\right) \subseteq C_{m-1}$ and (9) is true for $m \geq 2$ and $n=m-1<m$. Assume that (9) is valid for $n=k<m$. Note first that

$$
\begin{aligned}
\operatorname{co}\left(D_{m, k-1}\right) & =\operatorname{co}\left(\left\{z_{i}: k \leq i \leq m+1\right\}\right) \\
& =\operatorname{co}\left(\left\{z_{k}\right\} \cup\left\{z_{k+1}, \cdots, z_{m+1}\right\}\right) \\
& =\operatorname{co}\left(\left\{z_{k}\right\} \cup D_{m, k}\right) .
\end{aligned}
$$

Let $p \in \operatorname{co}\left(D_{m, k-1}\right)$ be given. If $p=z_{i}$ for some $i_{0} \in\{k, k+1, \cdots, m+1\}$, then $p \in C_{i_{0}} \subseteq C_{k-1}$. Suppose $p \neq z_{i}$ for all $i \in\{k, k+1, \cdots, m+1\}$. Thus, there exist $\gamma_{k}, \gamma_{k+1}, \cdots, \gamma_{m+1} \in[0,1)$ with $\sum_{i=k}^{m+1} \gamma_{i}=1$, such that $p=\sum_{i=k}^{m+1} \gamma_{i} z_{i}$. Let

$$
w=\sum_{i=k+1}^{m+1} \frac{\gamma_{i}}{1-\gamma_{k}} z_{i}
$$

Due to $\sum_{i=k+1}^{m+1} \frac{\gamma_{i}}{1-\gamma_{k}}=1$ and applying the induction hypothesis, we know

$$
w \in \operatorname{co}\left(D_{m, k}\right) \subseteq C_{k}
$$

and

$$
p=\sum_{i=k}^{m+1} \gamma_{i} z_{i}=\gamma_{k} z_{k}+\left(1-\gamma_{k}\right) w .
$$

Since $z_{k}, w \in C_{k}$, we have $f\left(z_{k}\right), f(w) \in \lambda_{k} e-K$ and hence

$$
\gamma_{k} f\left(z_{k}\right)+\left(1-\gamma_{k}\right) f(w) \in \lambda_{k} e-K .
$$

So $\gamma_{k} f\left(z_{k}\right)+\left(1-\gamma_{k}\right) f(w)=\lambda_{k} e-\beta$ for some $\beta \in K$. Since $\mu$ is $\precsim_{K}$-nondecreasing, by (H2), we obtain

$$
f(p) \precsim_{K} \mu\left(\gamma_{k} f\left(z_{k}\right)+\left(1-\gamma_{k}\right) f(w)\right)=\mu\left(\lambda_{k} e-\beta\right) \precsim_{K} \mu\left(\lambda_{k} e\right) \ll_{K} \lambda_{k-1} e
$$

which implies $p \in C_{k-1}$. Hence $c o\left(D_{m, k-1}\right) \subseteq C_{k-1}$. Therefore, (9) is true by mathematic induction. For any $n \in \mathbb{N}$, let

$$
U_{n}=\left\{x_{i}: i \geq n+1\right\} \text {. }
$$

Then $\operatorname{co}\left(U_{n}\right) \subseteq C_{n}$ for all $n \in \mathbb{N}$. Indeed, assume on the contrary that $\operatorname{co}\left(U_{j^{*}}\right) \nsubseteq C_{j^{*}}$ for some $j^{*} \in \mathbb{N}$. So, there exist $z_{k_{1}}, z_{k_{2}}, \cdots, z_{k_{s}} \in U_{j^{*}}$ and $\alpha_{1}, \alpha_{2}, \cdots, \alpha_{s} \geq 0$ with $\sum_{i=1}^{s} \alpha_{i}=1$, such that $\sum_{i=1}^{s} \alpha_{i} z_{k_{i}} \in \operatorname{co}\left(D_{k_{s}-1, k_{1}-1}\right)$ and $\sum_{i=1}^{s} \alpha_{i} z_{k_{i}} \notin C_{j^{*}}$. On the other hand, since $k_{i} \geq j^{*}+1$ for all $1 \leq i \leq s$, we have

$$
D_{k_{s}-1, k_{1}-1} \subseteq D_{k_{s}-1, j^{*}}
$$

and hence deduces from (9) that

$$
\operatorname{co}\left(D_{k_{s}-1, k_{1}-1}\right) \subseteq \operatorname{co}\left(D_{k_{s}-1, j^{*}}\right) \subseteq C_{j^{*}},
$$

which leads to a contradiction. Hence $c o\left(U_{n}\right) \subseteq C_{n}$ for all $n \in \mathbb{N}$. By the closedness of $C_{n}$, we get

$$
\overline{c o}\left(U_{n}\right) \subseteq C_{n} \text { for all } n \in \mathbb{N} .
$$


Since $\overline{c o}\left(U_{n+1}\right) \subseteq \overline{c o}\left(U_{n}\right)$ and $\overline{c o}\left(U_{n}\right)$ is weakly compact for all $n \in \mathbb{N},\left\{\overline{c o}\left(U_{n}\right): n \in \mathbb{N}\right\}$ is a family of closed subsets of the weakly compact set $\overline{c o}\left(U_{1}\right)$ which has the finite intersection property. Therefore we deduce

$$
\varnothing \neq \bigcap_{n \in \mathbb{N}} \overline{c o}\left(U_{n}\right) \subseteq \bigcap_{n \in \mathbb{N}} C_{n}
$$

and hence we can take $v \in \bigcap_{n \in \mathbb{N}} C_{n} \subseteq W$. So $F(v) \leq \lambda_{n}$ for all $n \in \mathbb{N}$. Since $\lambda_{n} \downarrow 0$ as $n \rightarrow \infty$, we get

$$
F(v) \leq 0 \Longleftrightarrow f(v) \in-K
$$

The proof is completed.

Corollary 3. Let $W$ be a nonempty weakly compact and convex subset of a normed linear space $(E,\|\cdot\|)$ with origin $\theta, \tau: \mathbb{R} \rightarrow \mathbb{R}$ be a nondecreasing function satisfying $\lim _{x \rightarrow 0^{+}} \tau(x)=0$ and $h: W \rightarrow \mathbb{R}$ be a function. Suppose that

(a) for any positive real number $\gamma,\{x \in W: h(x) \leq \gamma\}$ is a nonempty closed subset of $W$,

(b) $h$ is $(\tau)$-adjconvex.

Then there exists $v \in W$ such that $h(v) \leq 0$.

Proof. Take $Y=\mathbb{R}, K=[0,+\infty) \subset \mathbb{R}$ and $e=1$. Then $Y$ is a locally convex Hausdorff t.v.s. with its zero vector $\theta=0, K$ is a proper, closed and convex pointed cone in $Y$ with int $K=(0,+\infty) \neq \varnothing$, and $1 \in i n t K$. Define a partial ordering $\lesssim_{K}$ with respect to $K$ by

$$
x \precsim_{K} y \Longleftrightarrow y-x \in K .
$$

Then $h$ is a mapping from $W$ into $Y$ and $\tau: Y \rightarrow Y$ is a $\precsim_{K}$-nondecreasing function satisfying the condition (A) as in Lemma 3. Clearly, conditions (a) and (b) respectively imply conditions (H1) and (H2) as in Theorem 1. Hence all the assumptions of Theorem 1 are satisfied and therefore the desired conclusion follows immediately from Theorem 1.

As a direct consequence of Theorem 1, we obtain the following existence result.

Theorem 2. In Theorem 1, if the condition (H1) is replaced with conditions ( $h 1)$ and (h2), where

(h1) $f$ is $(e, K)$-lower semicontinuous;

(h2) for any positive real number $\gamma$, there exists $x \in W$ such that $f(x) \in \gamma e-K$.

Then there exists $v \in W$ such that $f(v) \in-K$.

Proof. For any positive real number $\gamma$, by (h1), (h2) and Lemma 2, the set

$$
\{x \in W: f(x) \in \gamma e-K\}
$$

is a nonempty closed subset of $W$. Therefore, the condition (H1) as in Theorem 1 holds. Applying Theorem 1, we can immediately obtain the conclusion.

Corollary 4. In Corollary 3, if the condition (a) is replaced with conditions (a1) and (a2), where

(a1) $h$ is lower semicontinuous;

(a2) for any positive real number $\gamma$, there exists $x \in W$ such that $h(x) \leq \gamma$.

Then there exists $v \in W$ such that $h(v) \leq 0$.

Applying Theorem 1, we can establish an existence theorem of zeros for vector-valued functions with $K$-adjustability convexity under an additional assumption. 
Theorem 3. In Theorem 1, if we further assume that $f(x) \in K$ for all $x \in W$, then the equation $f(x)=\theta$ has at least one root in $W$.

Proof. By Theorem 1, there exists $v \in W$ such that $f(v) \in-K$. Therefore, by our hypothesis, we get

$$
f(v) \in K \cap(-K)=\{\theta\},
$$

which deduces $f(v)=\theta$. Hence $v$ is a root of $f(x)=\theta$. The proof is completed.

As an immediate consequence of Theorem 3, we obtain the following new existence theorem.

Corollary 5. In Corollary 3 (or Corollary 4), if we further assume that $h(x) \geq 0$ for all $x \in W$, then the equation $h(x)=0$ has at least one root in $W$.

The following new existence and uniqueness theorem of zeros for vector-valued functions with strictly $(K, \mu)$-adjconvexity is established by applying Theorem 3.

Theorem 4. In Theorem 1, if we further assume $\mu(\theta)=\theta, f(x) \in K$ for all $x \in W$ and the condition (H2) is replaced with (H3), where

(H3) $f$ is strictly $(K, \mu)$-adjconvex,

then the equation $f(x)=\theta$ has a unique root in $W$.

Proof. Applying Theorem 3, the equation $f(x)=\theta$ has at least one root in $W$. Assume that $u, v \in$ $W$ are two distinct roots of $f(x)=\theta$. Since $W$ is convex and $\mu(\theta)=\theta$, we have $\frac{1}{2} u+\frac{1}{2} v \in W$ and $\mu\left(\frac{1}{2} f(u)+\frac{1}{2} f(v)\right)=\theta$. By $(\mathrm{H} 3)$, we get

$$
\mu\left(\frac{1}{2} f(u)+\frac{1}{2} f(v)\right)-f\left(\frac{1}{2} u+\frac{1}{2} v\right) \in \operatorname{int} K
$$

which implies

$$
f\left(\frac{1}{2} u+\frac{1}{2} v\right) \in-i n t K \cap K=\varnothing,
$$

a contradiction. Therefore, the equation $f(x)=\theta$ has a unique root in $W$. The proof is completed.

Corollary 6. In Corollary 3 , if we further assume that $\tau(0)=0, h(x) \geq 0$ for all $x \in W$ and the condition (b) is replaced with

(b1) $h$ is strictly $(\tau)$-adjconvex,

then the equation $h(x)=0$ has a unique root in $W$.

As an interesting application of Corollary 5, we prove the following minimization theorem.

Theorem 5. Let $W$ be a nonempty weakly compact and convex subset of a normed linear space $(E,\|\cdot\|)$ with origin $\theta$ and $g: W \rightarrow \mathbb{R}$ be a convex, lower semicontinuous and bounded below function. Then

$$
\arg \min _{x \in W} g(x):=\left\{y \in W: g(y)=\inf _{z \in W} g(z)\right\} \neq \varnothing .
$$

Moreover, if $g$ is strictly convex, then $\arg \min _{x \in W} g(x)$ is a singleton set.

Proof. Since $g$ is bounded below, $\inf _{z \in W} g(z)$ exists. Let $h: W \rightarrow \mathbb{R}$ be defined by

$$
h(x)=g(x)-\inf _{z \in W} g(z) \quad \text { for } x \in W .
$$


Clearly, the following hold:

- $h(x) \geq 0$ for all $x \in W$,

- $h$ is convex and lower semicontinuous.

Notice that for any $\gamma>0$, there exists $x_{\gamma} \in W$ such that $g\left(x_{\gamma}\right)<\inf _{z \in W} g(z)+\gamma$. Thus, we have

- $\quad$ For any positive real number $\gamma$, there exists $x \in W$ such that $h(x) \leq \gamma$.

Applying Corollary 5, there exists $v \in W$ such that $h(v)=0$, or equivalence, $g(v)=\inf _{z \in W} g(z)$. Hence $\arg \min _{x \in W} g(x) \neq \varnothing$. Assume that there exist $u, v \in \arg \min _{x \in W} g(x)$ with $u \neq v$. So $g(u)=g(v)=$ $\inf _{z \in W} g(z)$. Since $W$ is convex, we have $\frac{1}{2} u+\frac{1}{2} v \in W$. By the strict convexity of $g$, we get

$$
g\left(\frac{1}{2} u+\frac{1}{2} v\right)<\frac{1}{2} g(u)+\frac{1}{2} g(v)=\inf _{z \in W} g(z)
$$

which leads a contradiction. Therefore $\arg \min _{x \in W} g(x)$ is a singleton set. The proof is completed.

Finally, by applying Theorem 5, we establish a new fixed point theorem which is original and quite different from the well-known generalizations in the literature.

Theorem 6. Let $W$ be a nonempty weakly compact and convex subset of a normed linear space $(E,\|\cdot\|)$ with origin $\theta$ and $T: W \rightarrow W$ be a affine and continuous mapping. If $\inf _{x \in W}\|T x-x\|=0$, then $T$ admits a fixed point in $X$.

Proof. Define $g: W \rightarrow[0,+\infty)$ by

$$
g(x)=\|T x-x\| \quad \text { for } x \in W .
$$

Since $T$ is affine and continuous, $g$ is convex, continuous and bounded below function. By Theorem 5 , $\arg \min _{x \in W} g(x) \neq \varnothing$. Therefore, there exists $v \in W$ such that

$$
\|T v-v\|=g(v)=\inf _{z \in W} g(z)=\inf _{z \in W}\|T z-z\|=0 .
$$

Hence, we get $T v=v$. The proof is completed.

Remark 1. Theorems 1-6 and Corollaries 1-6 are completely original and quite different from the known results in the relevant literature.

\section{Conclusions}

The convexity of functions or sets plays a significant role in almost all branches of mathematics, physics, economics and engineering. In this paper, we introduce the concepts of $K$-adjustability convexity and strictly $K$-adjustability convexity which respectively generalize and extend the concepts of $K$-convexity and strictly $K$-convexity. Some new existence and uniqueness theorems of zeros for vector-valued functions with $K$-adjustability convexity are established. As their applications, we obtain existence theorems for minimization problem and fixed point problem which are original and quite different from the known results in the relevant literature.

Funding: This work was supported by Grant No. MOST 107-2115-M-017-004-MY2 of the Ministry of Science and Technology of the Republic of China.

Acknowledgments: The author wishes to express his hearty thanks to the anonymous referees for their valuable suggestions and comments.

Conflicts of Interest: The author declares no conflict of interest. 


\section{References}

1. Chen, G.Y.; Huang, X.X.; Yang, X.Q. Vector Optimization; Springer: Berlin/Heidelberg, Germany, 2005.

2. Chun, L.; Qi, F. Inequalities of Simpson type for functions whose third derivatives are extended s-convex functions and applications to means. J. Comput. Anal. Appl. 2015, 19, 555-569.

3. Hua, J.; Xi, B.-Y.; Qi, F. Inequalities of Hermite-Hadamard type involving an s-convex function with applications. Appl. Math. Comput. 2014, 246, 752-760. [CrossRef]

4. Komlósi, S. Generalized convexity and generalized derivatives. In Handbook of Generalized Convexity and Generalized Monotonicity; Hadjisavvas, N., Komlosi , S., Schaible, S., Eds.; Springer: New York, NY, USA, 2005; Volume 76, pp. 421-463.

5. Göpfert, A.; Tammer, C.; Riahi, H.; Zălinescu, C. Variational Methods in Partially Ordered Spaces; Springer: New York, NY, USA, 2003.

6. Hiriart-Urruty, J.-B.; Lemaréchal, C. Convex Analysis and Minimization Algorithms; Springer: Berlin/Heidelberg, Germany, 1993.

7. Hyers, D.H.; Isac, G.; Rassias, T.M. Topics in Nonlinear Analysis and Aapplications; World Scientific: Singapore, 1997.

8. Jahn, J. Vector Optimization Theory, Applications, and Extensions; Springer: Berlin/Heidelberg, Germany, 2011.

9. Luc, D.T. Theory of Vector Optimization, Lecture Notes in Economics and Mathematical Systems; Springer: Berlin/Heidelberg, Germany, 1989; Volume 319.

10. Luc, D.T. Generalized Convexity in Vector Optimization. In Handbook of Generalized Convexity and Generalized Monotonicity; Hadjisavvas, N., Komlosi, S., Schaible, S., Eds.; Springer: New York, NY, USA, 2005; Volume 76, pp. 195-236.

11. Qi, F.; Rahman, G.; Hussain, S.M.; Du, W.-S.; Nisar, K.S. Some inequalities of Čebyšev type for conformable $k$-fractional integral operators. Symmetry 2018, 10, 614. [CrossRef]

12. Rockafellar, R.T. Convex Analysis; Princeton University Press: Princeton, NJ, USA, 1970.

13. Shi, H.-N.; Du, W.-S. Schur-convexity for a class of completely symmetric function dual. Adv. Theory Nonlinear Anal. Appl. 2019, 3, 74-89.

14. Shi, H.-N.; Du, W.-S. Schur-power convexity of a completely symmetric function dual. Symmetry 2019, 11, 897. [CrossRef]

15. Zălinescu, C. Convex Analysis in General Vector Spaces; World Scientific: Singapore, 2002.

16. Gerth, C.; Weidner, P. Nonconvex separation theorems and some applications in vector optimization. J. Optim. Theory Appl. 1990, 67, 297-320. [CrossRef]

17. Chen, G.Y.; Huang, X.X. Ekeland? $\varepsilon$-variational principle for set-valued mappings. Math. Methods Oper. Res. 1998, 48, 181-186. [CrossRef]

18. Chen, G.Y.; Yang, X.Q.; Yu, H. A nonlinear scalarization function and generalized quasi-vector equilibrium problems. J. Glob. Optim. 2005, 32, 451-466. [CrossRef]

19. Du, W.-S. On some nonlinear problems induced by an abstract maximal element principle. J. Math. Anal. Appl. 2008, 347, 391-399. [CrossRef]

20. Du, W.-S. A note on cone metric fixed point theory and its equivalence. Nonlinear Anal. Theory Methods Appl. 2010, 72, 2259-2261. [CrossRef]

21. Du, W.-S. New cone fixed point theorems for nonlinear multivalued maps with their applications. Appl. Math. Lett. 2011, 24, 172-178. [CrossRef]

22. Du, W.-S. The existence of cone critical point and common fixed point with applications. J. Appl. Math. 2011, 2011, 985797. [CrossRef]

23. Du, W.-S.; Karapinar, E. A note on cone b-metric and its related results: generalizations or equivalence? Fixed Point Theory Appl. 2013, 2013, 210. [CrossRef]

24. Tammer, C. A generalization of Ekeland's variational principle. Optimization 1992, 25, 129-141. [CrossRef]

(C) 2019 by the authors. Licensee MDPI, Basel, Switzerland. This article is an open access article distributed under the terms and conditions of the Creative Commons Attribution (CC BY) license (http://creativecommons.org/licenses/by/4.0/). 